\title{
Características relevantes de grupos de investigación destacados en Andalucía
}

Mariana Altopiedi, Elena Hernández-de la-Torre y Julián López-Yáñez

\section{RESUMEN}

Este artículo pretende mostrar algunos resultados de una investigación sobre las características y la dinámica institucional de 101 grupos de investigación andaluces considerados de excelencia por el gobierno regional de Andalucía, España. ${ }^{1}$ El estudio se desarrolló en dos fases. La primera de ellas se basó en un cuestionario contestado por los responsables de 46 de esos 101 grupos de investigación destacados, y la segunda fase consistió en un estudio de casos múltiple. Presentamos los resultados relacionados con las características internas de estos grupos - composición, recursos humanos y materiales, dinámica social, estilo de funcionamiento, etcétera. El análisis y la interpretación de los resultados permiten caracterizar a los grupos de investigación andaluces en términos de su dinámica y del estilo de liderazgo predominante, así como la identificación de factores que condicionan el logro de la excelencia y la visibilidad en el campo académico en que cada uno se inserta. Permite, además, sugerir líneas de indagación que convendría explorar con mayor profundidad.

Palabras clave: grupos de investigación, excelencia académica, visibilidad en el campo científico, condicionantes de la labor investigadora, dinámica institucional, España.

\section{Mariana Altopiedi}

maltopiedi@us.es

Argentina/Española. Doctora en Ciencias de la Educación por la Universidad de Sevilla, España. Profesora Titular de la Escuela Universitaria de Osuna, adscrita a la Universidad de Sevilla. Temas de investigación: innovación educativa y procesos de cambio y mejora, asesoramiento educativo, dirección y liderazgo en organizaciones educativas, desde la perspectiva del análisis institucional y la investigación narrativa.

Elena Hernández de la Torre

eht@us.es

Española. Doctora en Ciencias de la Educación por la Universidad de Sevilla, España. Profesora Titular en la Facultad Ciencias de la Educación y el Departamento de Didáctica y Organización Educativa. Temas de investigación: atención a la diversidad y escuela para todos, innovación y mejora para la formación de profesores y profesionales, y función tutorial del profesor universitario para el desarrollo de buenas prácticas docentes.

Julián López Yáñez

lopezya@us.es

Español. Doctor en Ciencias de la Educación por la Universidad de Sevilla. Profesor titular del Departamento de Didáctica y Organización Educativa de la Universidad de Sevilla. Temas de investigación: liderazgo, dinámicas institucionales de cambio, conocimiento y aprendizaje organizativo, y teoría de la organización en organizaciones educativas.

\footnotetext{
${ }^{1}$ López Yáñez, Julián (coord.) (2008-2010) "El poder de las redes sociales. Análisis institucional de grupos de investigación de excelencia", proyecto financiado por la Junta de Andalucía en la convocatoria de Proyectos de Investigación de Excelencia (referencia: P07.SEJ-02960).
} 


\title{
Características relevantes de grups de pesquisa destacados em Andaluzia
}

\section{RESUMO}

Este artigo pretende mostrar alguns resultados de uma pesquisa sobre as características e a dinâmica institucional de 101 grupos de pesquisa andaluzes considerados de excelência pelo governo regional da Andaluzia, Espanha. O estudo foi desenvolvido em duas fases. A primeira baseou-se num questionário respondido pelos responsáveis de 46 desses 101 grupos de pesquisa destacados, e a segunda fase consistiu num estudo de casos múltiplos. Apresentamos os resultados relacionados com as características internas desses grupos (composição, recursos humanos e materiais, dinâmica social, estilo de funcionamento, etecetera). A análise e a interpretação dos resultados permitem caracterizar os grupos de pesquisa andaluzes em termos da sua dinâmica e do estilo de liderança predominante, bem como a identificação de fatores que condicionam o sucesso da excelência e a visibilidade no campo acadêmico em que cada um deles se insere. Permite, aliás, sugerir linhas de indagação que conviria explorar com maior profundidade.

Palavras chave: grupos de pesquisa, excelência acadêmica, visibilidadeno campo científico, condicionantes do trabalho de investigação, dinâmica institucional, Espanha.

\section{Relevant characteristics of distinguished research groups in Andalusia}

\begin{abstract}
This article tries to show some of the results of research work about the characteristics and institutional dynamics of 101 Andalusian research groups considered as group of excellence by the regional government of Andalusia, Spain. The study was developed in two stages. The first one was based on a questionnaire answered by thepersons responsible for 46 out of these 101 outstanding research groups, and the second stage consisted of a study of multiple cases. We present the results related to the internal characteristics of said groups: composition, human and material resources, social dynamics, operation style, etc. The analysis and interpretation of the results enabled the characterization of the Andalusian research groups in terms of their dynamics and predominant leadership style, as well as the identification of factors that determine the achievement of excellence and the visibility in each of their academic fields. It also suggests lines of research that should be explored in greater detail.
\end{abstract}

Key words: research groups, academic excellence, visibility in the scientific field, determinants of research work, institutional dynamics, Spain.

Recepción: 07/07/13. Aprobación: 06/02/15. 


\section{Los grupos de investigación de excelencia y sus características: aportaciones de la literatura}

Es evidente que la ciencia ha alcanzado una importancia decisiva en la sociedad del conocimiento. Como consecuencia, el estudio de los procesos de producción y distribución del conocimiento científico, así como "de aquellos problemas que afectan a las organizaciones y a los científicos que producen ciencia y tecnología" se ha convertido en objeto de interés creciente (Fernández y Torres, 2009: 665). Uno de los ámbitos destacados de este campo de estudio es el que pone el foco sobre los grupos de investigación, en tanto que constituyen el principal contexto de socialización profesional de los científicos (Carayol y Matt, 2004; Rey-Rocha et al., 2006; 2008; Boardman y Corley, 2008; Horta y Lacy, 2011 ; Blasi y Romagnosi, 2012). Es fundamentalmente a partir de las interacciones que tienen lugar en el marco de estos grupos que las nuevas generaciones de científicos incorporan e interiorizan los modos de hacer, los valores y significados culturales, y las reglas de cada disciplina o campo científico (Blasi y Romagnosi, 2012), al tiempo que contribuyen a su transformación.

Sin embargo, la revisión de la producción teórica y empírica sobre este ámbito de estudio revela su carácter controvertido, y ello tanto por la delimitación de la unidad de análisis - grupos de investigación versus académicos, considerados individualmente- como por la definición misma del concepto de grupo o equipo de investigación. En este sentido, según Rey-Rocha et al. (2008), cabe diferenciar tres aproximaciones:

- En razón de los logros comunes y las producciones conjuntas de un conjunto de científicos, o output-based studies.

- En razón de los insumos o recursos compartidos por un conjunto de científicos, o input-based studies.

- En razón de una identidad o sentido de afiliación compartidos y creados en el marco de una trayectoria común. Es decir, se trataría de estudios fundados sobre la percepción que el propio grupo tiene de sí mismo como tal.

Esta última aproximación subraya la dimensión informal de las relaciones entre sus miembros, su carácter estable y el hecho - vinculado a éste- de que van desarrollándose a lo largo del tiempo. Así, en su continuidad son capaces de transformarse para buscar formas de operar adaptadas a los cambios en el contexto (Sundstrom et al., 1990). Paradójicamente, la estabilidad los vuelve flexibles en tanto asumen una dinámica progresiva (Bleger, 1994) orientada a resolver racionalmente las dificultades, que alterna con momentos de estancamiento o de regresión.

En este estudio, los grupos de investigación se seleccionaron siguiendo el segundo criterio, de tal manera que identificamos grupos que compartían un conjunto de recursos y eran reconocidos oficialmente como tales dentro del Plan Andaluz de Investigación, Desarrollo e Innovación (PAIDI). Los grupos participantes se seleccionaron mediante la consulta al organismo del cual dependían, la Junta de Andalucía.

Uno de los principales fines de los estudios sobre grupos de investigación es identificar factores susceptibles de explicar las diferencias en su productividad. Los usualmente contemplados son:

- Factores contextuales

- Área de conocimiento

- Ámbito geográfico y organizativo de inserción

- Fuentes de financiamiento

- Prestigio de la organización

- Características internas del grupo

- Tamaño, atendiendo a la cantidad de miembros o a la representación de distintas categorías profesionales o académicas

- Composición, más o menos homogénea o bien multidisciplinar

- Niveles de cualificación de los miembros, asociados a los mecanismos de selección, 
predictores de la productividad (Prpic, 1994)

- Vinculación de los miembros con la organización (pertenencia formal, tipo de dedicación y modalidad de contratación, etcétera)

- Cohesión o integración social/ formas de colaboración en la tarea

- Liderazgo, considerado como movilizador de la creatividad y la productividad mediante la generación de un clima adecuado (Hemlin, 2006)

- Características personales de sus miembros

- Edad, cuya repercusión está asociada al campo de conocimiento

- Sexo, aparentemente menos influyente

- Personalidad, en particular la tolerancia a la exigencia y la presión

- Experiencia dentro del campo, asociada al grado o cualificación

En este artículo nos centramos en el segundo tipo de factores, en las características internas del grupo que inciden en la labor académica de sus miembros, condicionando su productividad y capacidad de destacar en el campo científico del que forman parte. Concretamente, indagamos en el modo en que factores de orden estructural - tales como la cantidad y la estabilidad de los miembros o la pertenencia institucional de los grupos - así como de tipo dinámico — relativos a la organización de la tarea académica y las relaciones de poder que contribuyen a configurarla-, influyen en el rendimiento y la visibilidad de estos grupos.

Este interés obedece fundamentalmente a la convicción de que, dado el carácter vocacional de la actividad científica y el elevado grado de exigencia que supone su desempeño, ésta exige un alto nivel de motivación (Hackett, Conz, Parker, Bashford y DeLay, 2004) así como un marco socioemocional que la haga sostenible. En este sentido, coincidimos con Hoffman y otros (2013) en la necesidad de una mejor comprensión de las dinámicas sociales de estos grupos, en tanto es la principal condición organizativa que afecta al rendimiento científico.

La importancia concedida a estas cuestiones está también en la base del "giro institucional" que se ha venido produciendo en la sociología de la ciencia (Fernández y Torres, 2009), orientando cada vez más el estudio de los procesos de producción del conocimiento hacia las organizaciones y los grupos dedicados a ello, en detrimento de los científicos y sus trayectorias, tomados individualmente. Desde esta perspectiva se presta especial consideración a las condiciones tanto internas - vinculadas a la dinámica de cada colectivo de académicos - como relativas a sus relaciones con el resto de la sociedad, que afectan a la labor científica. Disminuye así la atención dada a cuestiones tales como la historia de la ciencia y los rasgos que la definen como actividad y como institución social, así como el interés por los procedimientos concretos de generación y validación del conocimiento científico.

Por otro lado, las características de los grupos científicos a las que prestamos atención en este artículo no son estáticas, sino que cambian a lo largo de su trayectoria (Tuckman, 1965). Esto justifica la adopción de una perspectiva dinámica que contemple a los grupos como "entidades dinámicas sujetas a ciclos evolutivos" (Rey-Rocha et al., 2008: 751). Dicha evolución depende de múltiples factores, tanto intrínsecos como contextuales (ibid.) y en su trayectoria pueden distinguirse etapas, por ejemplo las planteadas inicialmente por Tuckman y luego desarrolladas por él mismo y sus colaboradores (Tuckman y Jensen, 1977; Rickards y Moger, 2000): formación, confrontación, regulación, desempeño y renovación (forming, storming, norming, performing, adjourning). Si bien este artículo no adopta una perspectiva evolutiva en el análisis de los resultados de la investigación en la que se basa, sí que hemos tratado de identificar algunas de las características de estos grupos que condicionan su evolución y, más concretamente, aquellas que comprometen sus posibilidades de alcanzar visibilidad y notoriedad en su campo científico. 


\section{Enfoque metodológico y contexto del estudio}

El diseño de investigación combinó una fase cuantitativa y exploratoria con otra de estudio de casos mediante estrategias de corte cualitativo. La puesta en juego de ambos enfoques se funda en la convicción de que pueden complementarse, si se parte de un marco axiológico y epistemológico común. En nuestro caso, se trata del análisis institucional de los grupos y de las organizaciones, caracterizado por ser interpretativo y concentrarse en el modo en que los sujetos construyen el marco social, institucional y organizativo del que participan, al tiempo que son constituidos por él.

Desde la perspectiva cuantitativa y descriptiva se aplicó un cuestionario diseñado ad hoc, dirigido a los responsables de grupos de investigación de excelencia, reconocidos por la entonces Consejería de Innovación y Ciencia. Este organismo, encargado de la promoción de la investigación en la Comunidad Autónoma, reconoce a las unidades dedicadas a la investigación y, en función de la periódica evaluación de su producción, les asigna fondos y reconocimientos, entre los que se halla la consideración de su "excelencia".

El cuestionario fue diseñado en un proceso de sucesivas reformulaciones, supervisado por revisores externos, a partir de cuestiones reseñadas en la literatura para la caracterización de los grupos científicos. El diseño adoptado finalmente constaba de cuatro apartados relacionados con la composición del grupo, su organización material, su funcionamiento interno y sus relaciones con otras organizaciones, así como con posibles problemas y dificultades.

En cada apartado se formulaban preguntas cerradas de distinto formato. Finalmente, se incluían dos preguntas abiertas, sobre la historia y la proyección del grupo. Si bien la mayoría de los participantes contestó el total de las preguntas cerradas, no se registraron respuestas a las de formato abierto.
La administración del cuestionario se realizó electrónicamente, invitando a los directores de los 101 grupos de investigación registrados por la administración andaluza. Después de dos meses y varios recordatorios, se recogieron 46 respuestas válidas, en cuyo análisis se basan las interpretaciones que expondremos.

El tratamiento estadístico de los datos - estrictamente descriptivo - se realizó mediante el paquete SPSS 11.0. Intencionadamente, se renunció al establecimiento de correlaciones, atendiendo al limitado número de respuestas y al enfoque de estudio, que eludía la formulación de generalizaciones.

Desde el punto de vista cualitativo e interpretativo, se empleó la metodología de estudio de casos, considerando diversos criterios en la selección de éstos. En primera instancia, se atendió a su relevancia en términos de las subvenciones recibidas y de la visibilidad de su producción - medida en función de publicaciones, premios y repercusión social. En segundo lugar, se procuró la representación de distintas áreas de conocimiento, en atención a la literatura relativa a las peculiaridades de las distintas ramas científicas o "tribus académicas" (Becher, 2001). Se seleccionaron así cinco casos, representando a las áreas Científica, Humanidades, Ciencias Jurídicas y Sociales, Tecnológica y de Ciencias de la Salud. Finalmente, se consideró también la pertenencia de estos grupos a organizaciones diversas, tanto universitarias como dedicadas exclusivamente a la investigación.

En los estudios de casos se realizaron entrevistas semiestructuradas, usando un guión diseñado a partir de los resultados obtenidos en la primera fase y de las dimensiones propuestas en la literatura para caracterizar los grupos científicos y su dinámica. Éste contemplaba cuestiones relativas a la inscripción organizativa del grupo, atendiendo a su acceso a los recursos; su posición institucional y su vinculación con el entorno, indagando en sus relaciones con la institución de referencia, así como con otras organizaciones y grupos 
o redes; la tarea o práctica cotidiana; los miembros y los tipos de trayectorias que mostraban; la historia institucional del grupo; la dinámica social, considerando la influencia relativa de los miembros y sus fuentes de poder; la cultura organizativa o conjunto de ideas, concepciones, maneras de pensar y de actuar, que conforman el estilo de funcionamiento grupal; la disciplina científica de referencia y su ubicación en el panorama académico general y en nuestro país.

Asimismo, se llevaron a cabo observaciones orientadas a captar el funcionamiento cotidiano de los grupos mediante una estrategia de "marcaje" (shadowing) de los investigadores, consistente en el acompañamiento de algunos de ellos a lo largo de su jornada laboral, durante entre uno y tres días, en función de las posibilidades y disponibilidad de cada investigador participante. Se procedió también al análisis de sus páginas web, a fin de obtener información relativa tanto a sus relaciones con el entorno - políticas de difusión del trabajo - como a su organización interna, reflejada en la presencia de los distintos miembros en ellas. Asimismo, se rastreó el impacto de su trabajo en términos de publicaciones y de su repercusión a través de la recopilación de menciones a su actividad en periódicos o publicaciones no especializadas, identificación de sus actividades de extensión cultural, etcétera.

Dados el tamaño y la dispersión geográfica de algunos de los grupos de investigación, no todos sus miembros pudieron participar en el estudio. En cualquier caso, siempre se garantizó la participación del director o líder y del fundador del grupo, así como de miembros representativos de cada generación académica. En total, entre cinco y 11 miembros de cada grupo participaron del estudio, representando entre el $30 \%$ y el $100 \%$ de los integrantes de cada uno. Además, en los casos en que esto fue posible y de manera complementaria, se entrevistó a miembros de otros grupos de investigación inscritos en la organización, así como a un directivo de la misma.
Dada la amplitud de la información obtenida a través de las distintas estrategias y técnicas de investigación puestas en juego, nos centramos aquí en la devenida de los datos de carácter cuantitativo, ampliados en las ocasiones en que resulta pertinente con ilustraciones derivadas de la interpretación de los datos cualitativos. De igual modo, a fin de delimitar el objeto de este artículo, nos centramos por razones de orden y espacio - en algunos de los aspectos indagados. Concretamente, presentaremos las interpretaciones relativas a las características internas de los grupos de investigación analizados.

\section{Algunos hallazgos relevantes de la investigación}

Atendiendo a los fines y al enfoque de la investigación, así como a los resultados de estudios previos, interesaba tanto indagar aspectos de orden estructural - como el tamaño y la pertenencia de los gruposcomo otros de carácter dinámico, entre los que se incluían las configuraciones de poder y los estilos de liderazgo según son representados por los integrantes. A continuación se exponen algunos de los resultados obtenidos en relación con estas cuestiones que nos parecen especialmente significativos. Como podrá verse, muchos de ellos tienden a confirmar hallazgos de estudios previos - que comentaremos en relación con cada aspecto abordado - mientras que otros evidencian un claro contraste con ellos.

\section{Trayectoria, flujo de investigadores $y$ estabilidad del grupo}

De acuerdo con los datos, los grupos de investigación andaluces de excelencia cuentan con una trayectoria breve, que no supera una década en, aproximadamente, un tercio de ellos. Esto debería matizarse contemplando su posible funcionamiento anterior a su creación oficial, asociada al comienzo de las convocatorias para la constitución de los "grupos PAI". 2 Algunos de los grupos "fundados" entonces ya trabajaban como tales anteriormente, aunque de 
un modo no institucionalizado ni reconocido administrativamente debido a la ausencia de organismos de carácter oficial encargados de ello. Refuerza esta idea la recurrencia con la que varios de estos grupos fueron valorados como "de excelencia" por la Consejería competente, en sus evaluaciones anuales.

Quizá por su relativa juventud, la dirección de la mayoría de los grupos era ejercida, al recolectarse los datos, por la misma persona que al fundarse (33 casos). En nueve de ellos había habido dos directores y sólo cuatro grupos habían tenido tres.

Una relativa excepción la constituye uno de los grupos participantes en el estudio de casos, donde se verificaba el movimiento de la investigadora principal a otra organización en al menos tres ocasiones a lo largo de su trayectoria académica. Ello exigía un cambio de titularidad en la dirección del grupo matriz que, pese a ello, se mantenía vinculado al nuevo grupo creado por la mencionada investigadora. De este modo, la fundadora seguía ejerciendo un papel de autoridad - informal pero importante - en los grupos de los que administrativamente se había desvinculado. Sus sucesivos traslados suponían la simultánea apertura de espacios académicos en la nueva organización y la posibilidad de promoción de sus colaboradores en la organización que dejaba. Mediante esta cesión de la autoridad - que define una estrategia con claras similitudes con la que Blasi y Romagnosi (2009) denominan "colonialista" - su liderazgo se veía simbólicamente fortalecido en tanto aumentaba su imagen de grandeza personal.

Desde nuestro punto de vista, el hecho de que la mayoría de los grupos científicos analizados se constituyeron como tales en torno a un académico prestigioso establece una configuración a modo de pequeño imperio donde el mecanismo habitual de incorporación es la solicitud de los interesados y a continuación la colaboración sostenida. Quienes desean integrar el grupo comienzan contribuyendo de manera externa y voluntaria, hasta ser oficialmente admitidos tras cierto periodo de prueba.

Esta disposición se hace patente en nuestros estudios de caso. Uno de los grupos, del ámbito universitario, cuenta con la colaboración de becarios que anteriormente se desempeñaron como alumnos internos y colaboradores honorarios. Otro, ajeno a la universidad, ha establecido con ella un convenio para recibir alumnos en prácticas, aspirantes a integrar el grupo. Una vez formados y socializados en el habitus académico propio del grupo, algunos de estos miembros jóvenes que han logrado ser reconocidos como parte del grupo pero por razones administrativas o ante la falta de dotación económica no pueden permanecer en él, son "enviados a conquistar" otras organizaciones, siguiendo una estrategia de difusión de las ideas del grupo próxima a la de "extroversión" que plantean Blasi y Romagnosi (2009).

También Etzkowitz (1993) describe la constitución de los grupos de investigación como un proceso de agrupamiento en torno de un investigador principal - generalmente, con alto rango académico y prestigioso en su campo-, de colegas o colaboradores que los académicos de mayor experiencia se encargan de formar. Una vez conformados, estos grupos compiten por financiamiento y reconocimiento simbólico, en un proceso de paulatina diferenciación respecto del marco organizativo - el departamento universitario, por ejemplo - con el consiguiente establecimiento de fronteras (Sundstrom et al., 1990) entre un "adentro" y un "afuera". En la medida en que van obteniendo logros, los grupos se consolidan, alcanzando una relativa estabilidad. La incorporación de nuevos miembros que suplantan a aquellos que - por independizarse en su actividad investigadora o por retirarse del ámbito académico - se separan de él, hace posible, simultáneamente, la renovación y la continuidad del grupo.

\footnotetext{
${ }^{2}$ Plan Andaluz de Investigación, surgido como mecanismo para impulsar las acciones de I+D+i en la Comunidad Autónoma de Andalucía, tras la transferencia de las competencias en este ámbito desde el estado nacional a las administraciones de cada una de las fracciones en las que se organiza políticamente el territorio español.
} 
En los últimos tiempos y en parte debido al proceso de marketización (Lucas, 2006) —la sobrevaloración de la capacidad para atraer financiamiento - este mecanismo de sucesión generacional parece verse obstruido. Los recortes en las aportaciones económicas de origen público y el endurecimiento de las condiciones de profesionalización en el campo académico obstaculizan la promoción de los investigadores jóvenes, poniendo en riesgo la continuidad de los grupos que perciben - como veremos luegoesta amenaza.

A lo largo de su historia, puede identificarse una evolución de los grupos desde un trabajo compartido algo asistemático y espontáneo, que cabe equiparar al que tiene lugar durante las fases de formación y confrontación (Tuckman y Jensen, 1977; Rickards y Moger, 2000), hacia la progresiva consolidación e institucionalización (regulación y desempeño, en términos de estos autores). Esta fase es seguida por otra de desintegración, fragmentación o desmembramiento total o parcial... y eventual renovación (Tuckman y Jensen, 1977; Rickards y Moger, 2000). Estas fases de mayor o menor integración y productividad son condicionadas tanto por la propia dinámica como por variables personales, inherentes a los miembros, y por factores contextuales.

Entre estos últimos cabe señalar la pertenencia organizativa de los grupos de investigación que, según los datos, no ha variado para los grupos de la muestra. Como se aprecia en la gráfica 1, la mayoría de ellos pertenece a organizaciones universitarias.

Dado que estudios como el de Horta y Lazy (2011) muestran que el tamaño total del grupo medido en cantidad de integrantes guarda escasa relación con la productividad del conjunto, a la hora de caracterizar a los grupos de investigación participantes en nuestra investigación según su tamaño adoptamos el criterio de considerar la cantidad de miembros estables, que asumen la mayor variedad y cantidad de tareas de docencia y gestión complementarias a la investigación. El análisis de las respuestas al cuestionario permite afirmar que los grupos de excelencia andaluces tienen un tamaño relativamente pequeño aunque existe cierta polarización. Mientras el $44.2 \%$ de los grupos tiene seis investigadores estables o menos, otros ocho superan los 15 miembros permanentes. Son escasos los que cuentan con entre diez y 15 investigadores estables, como puede observarse en la gráfica 2.

\section{Gráfica 1. Centro u organismo de pertenencia}

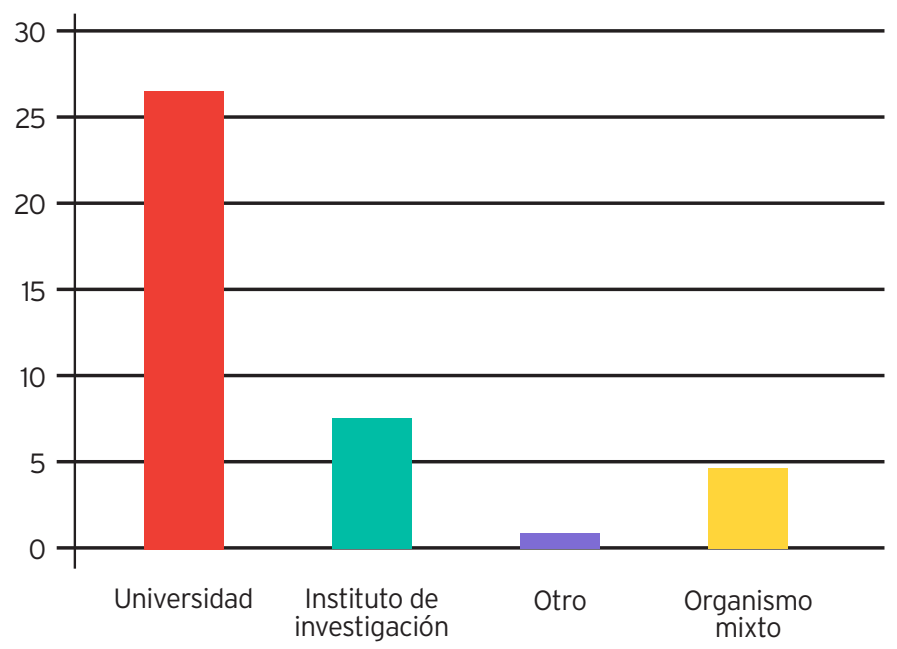


La cantidad de investigadores invitados o no estables es notoriamente inferior a la de miembros permanentes, y las labores que realizan suelen limitarse a la investigación, en la que su participación es - según nuestros datos - del mismo carácter que la de los investigadores estables. Por su parte, los investigadores en formación - pre o posdoctoral- son menos numerosos: no más de seis, en la mayoría de los casos, como se aprecia en las gráficas 3 y 4 .

\section{Gráfica 2. Investigadores estables en el grupo}

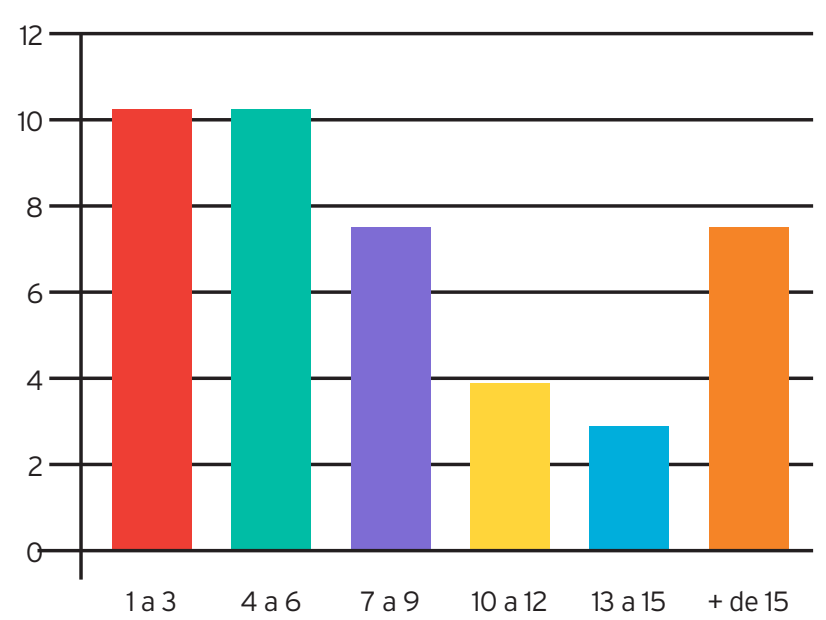

La mayor parte de estos investigadores no asumen tareas de docencia ni de gestión; generalmente se encuentran elaborando su tesis doctoral o desarrollando los proyectos que les valieron el disfrute de una beca o contrato de formación. Dada la reiterada mención a las dificultades para estabilizar a estos miembros en el grupo, creemos que la concentración de sus esfuerzos en labores académicamente rentables responde, por otro lado, a un intento - tanto

\section{Gráfica 3. Investigadores posdoctorales}

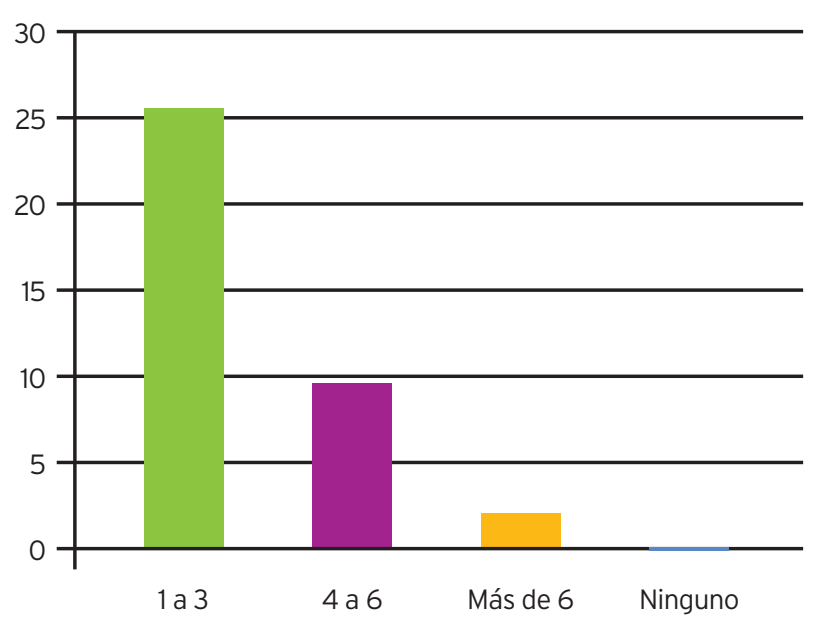

\section{Gráfica 4. Becarios predoctorales}

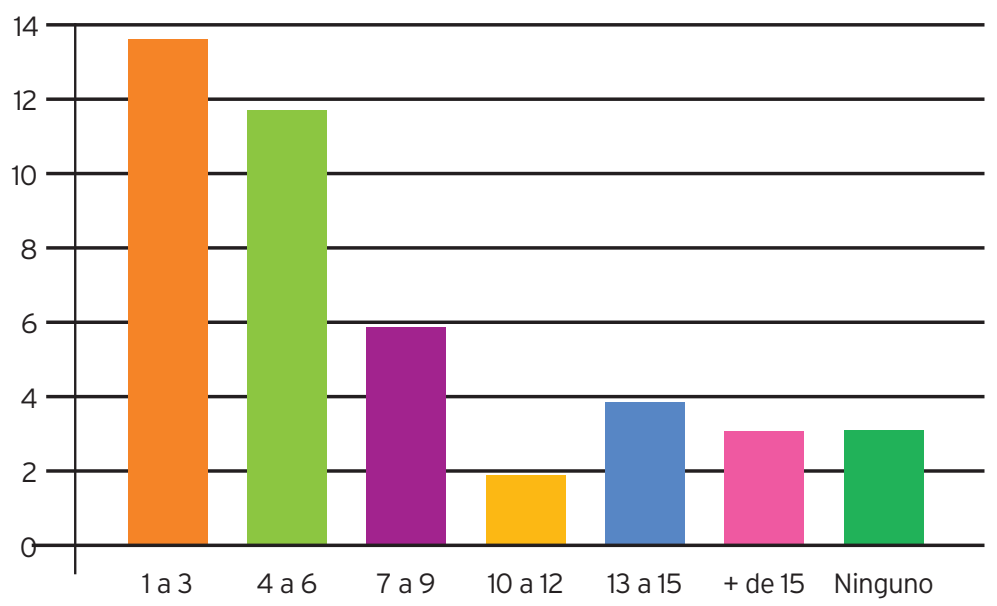


individual como colectivo- de contrarrestar estos obstáculos. El empleo de esta estrategia contribuye, asimismo, a explicar la positiva incidencia que la literatura atribuye a la presencia de becarios y personal contratado en formación, en la productividad de los grupos de investigación (Triadó y Aparicio, 2006).

En síntesis, se identifica una clara distribución de roles y funciones entre miembros estables, invitados y en formación, favorable a todos y al conjunto. A través de compartir procedimientos y formas de trabajo, cada grupo va conformando un estilo característico que se transmite a las siguientes generaciones académicas. Siendo gran parte de este bagaje tácito y experiencial, se transfiere a través de mecanismos informales, como la participación en prácticas compartidas que opera en la socialización de los nuevos miembros de la "tribu académica" (Becher, 2001). En esta interacción se pone en juego el saber hacer profesional que define el habitus de cada ámbito del conocimiento (Bourdieu, 2007) y los participantes van apropiándose de este conjunto de disposiciones y saberes más o menos formalizados, asegurando su mantenimiento.

Simultáneamente, operan profundos mecanismos de identificación, que aseguran cierta compenetración emocional y la adopción de pautas de acción comunes o complementarias. Paralelamente al proceso de organización formal, van conformándose formas de actuar, sentir y significar la realidad características de cada grupo. A medida que van consolidándose y el grupo adquiere estabilidad, comienzan a manifestarse diferencias que, en lugar de suponer un riesgo para la cohesión grupal, son valoradas como aportación al conjunto. Esto permite que asuman otras dos funciones de integración que, siguiendo a Moreland (1987), señalan Rey-Rocha et al. (2008): ambiental, relacionada con el espacio - físico y simbólico - que comparten y conductual. Esta última forma de integración se vincula con la relativa dependencia entre los miembros en tanto comparten objetivos y recursos.

\section{Organización del grupo y del trabajo científico}

Como puede verse en la gráfica 5, los grupos de investigación estudiados se organizan mayoritariamente "en subgrupos definidos en subáreas o intereses académicos compartidos", según afirma el $73.9 \%$ de los participantes. Un $17.4 \%$, por su parte, sostiene que "cada investigador trabaja de manera autónoma, con el apoyo ocasional de otros miembros"; un 13\% afirma que "cada investigador trabaja de manera autónoma, con apoyo de becarios y/o colaboradores"; y un $8.7 \%$ señala que el grupo completo trabaja en cada actividad. Desde nuestro punto de vista, este modo de operar está vinculado con la comentada polarización en el tamaño de los grupos. Sólo en los grupos más reducidos la colaboración entre todos los miembros parece posible. En los de mayor tamaño, la conformación de múltiples subgrupos relativamente autónomos facilita la tarea y evita desavenencias. En cambio, la conformación de estas "micro-unidades de trabajo" genera en ocasiones dificultades a los grupos pequeños, donde algunos miembros, especialmente los recién llegados, pueden verse aislados.

Por otra parte, los grupos de investigación de nuestro estudio tendieron a adoptar una forma de trabajo basada en el consenso y en la libre adscripción de sus miembros a subgrupos constituidos en función de intereses académicos compartidos. Las afinidades personales, en cambio, carecían de relevancia en los patrones de colaboración. Esto resulta congruente con lo observado en los estudios de caso, dado que estos grupos aparecen centrados en la tarea y disponen de los mecanismos de cohesión social suficientes para que los aspectos afectivos no la perturben. En este sentido, es frecuente la organización - más o menos sistemática y regular - de actividades sociales que congregan a los miembros, con independencia de su lugar en el grupo.

Por lo demás, siendo que estos grupos se constituyen por el acercamiento de uno o más investigadores 


\section{Gráfica 5. Forma habitual de organización del trabajo científico}

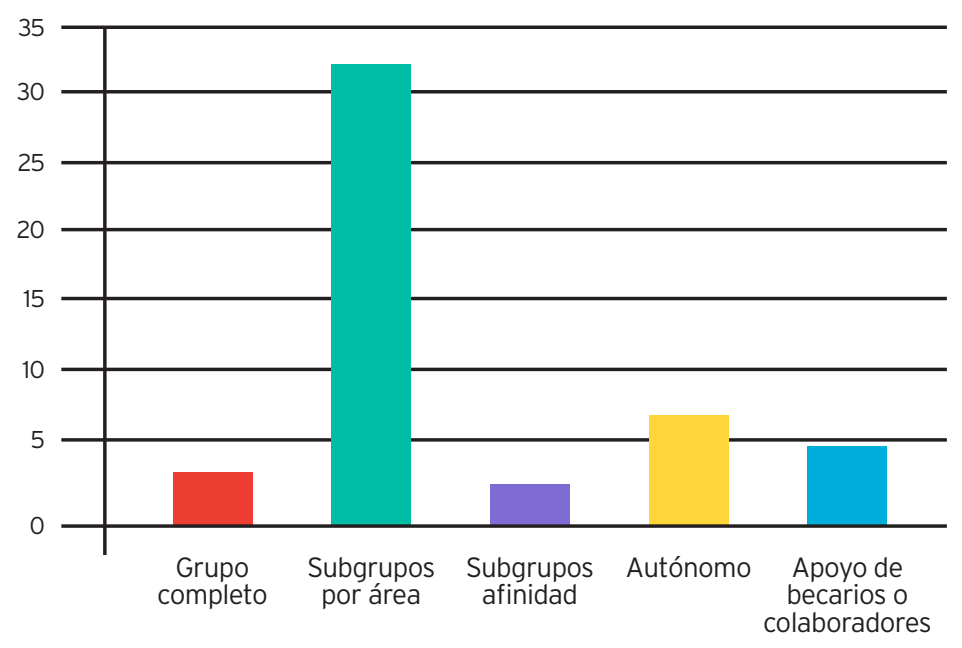

en formación a un investigador experimentado, la disparidad en cuanto a edad, experiencia vital y situación profesional limita la posibilidad de coincidencias en gustos e intereses personales. Como señalara el investigador principal de uno de los casos estudiados, la diferencia de edad con respecto a los demás miembros del grupo le permite verlos como "hijos". Como contrapartida, estos investigadores jóvenes tienden a tomar al director del grupo como modelo intelectual y objeto de admiración. Similarmente, en otro caso, la directora del grupo es nombrada - incluso por sus colaboradores más antiguos y cercanos - con el respetuoso y formal tratamiento de "Doña". Sin duda los mecanismos socioafectivos de búsqueda del reconocimiento de una figura a la que se admira, visibles detrás de estas manifestaciones, contribuyen a incrementar el nivel de motivación de los jóvenes investigadores. Si, como señalan Hackett et al. (2004), este sentimiento es necesario para implicarse en una tarea exigente, emocional e intelectualmente, como la académica, más aún lo es en las precarizadas condiciones de trabajo actuales, donde los plazos para que estos investigadores obtengan reconocimiento y una posición estable, se alargan.

$\mathrm{Al}$ no contar con suficiente autonomía para elegir un problema de investigación propio, los investigadores noveles suelen iniciarse en alguno subordinado al de otro miembro del grupo. ${ }^{3}$ Paradójicamente, esto favorece la ampliación del horizonte de la investigación originaria, haciendo que se diversifique y adquiera nuevos matices. La labor de estos investigadores en formación es, entonces, una vía para la diseminación y el crecimiento de la línea de indagación que, de este modo, va ganando visibilidad y legitimándose. Tal es el caso de la línea de investigación en museología surgida en uno de los grupos estudiados, a raíz tanto de la necesidad de atender a nuevas cuestiones surgidas en su campo - la arqueología - como del virtual agotamiento de líneas exploradas por investigadores de mayor trayectoria.

\footnotetext{
${ }^{3}$ Interesa atender, en este aspecto, a las aportaciones de autores como Bourdieu (2007) y Ziman (1987) al reconocimiento del papel de las múltiples relaciones de poder actuantes en el ámbito académico a la hora de seleccionar líneas o problemas de investigación.
} 
Por su parte, la distribución de líneas de investigación entre investigadores que mantienen una relación de iguales se produce por acuerdo, permitiendo cierta autonomía y respetando intereses, conveniencias y/o gustos personales.

La caracterización precedente permite afirmar que los grupos estudiados tienen un funcionamiento participativo y relativamente democrático. Esta visión responde a las opiniones de los directores y, probablemente, pueda limitarse a algunos aspectos del funcionamiento del grupo, así como a un sector de sus componentes. Sin embargo, no alcanza del mismo modo a los miembros más jóvenes o menos prestigiosos.

En ese sentido, los datos evidencian que la toma de determinadas decisiones relevantes se centraliza en quien dirige al grupo. En muchos casos también se concentran en los investigadores principales las tareas de gestión - incluyendo en buena medida las administrativas -, las cuales requieren importantes esfuerzos que no son de inmediato provecho para quien los realiza. Coexiste, en definitiva, la tendencia a la centralización de algunas decisiones en el director del grupo - por ejemplo, la convocatoria a los miembros - junto con mecanismos de consenso en la distribución de funciones y tareas. La elección de uno u otro estilo de funcionamiento obedece a necesidades del momento.

Por otro lado, la literatura especializada atribuye a los líderes de grupos de investigación múltiples funciones, centrales en el impulso del funcionamiento grupal. Según el modelo de Latour (2001) el directivo debe atender a cinco "bucles" interrelacionados que participan en la circulación de los hechos científicos, que atañen a aspectos tan heterogéneos como: la puesta en juego de recursos; el establecimiento de alianzas para la conformación de la ciencia como campo autónomo y del propio grupo en su disciplina; la representación pública de la ciencia; la conformación de los vínculos entre los miembros, así como la conformación de los nudos conceptuales que dan sentido a los otros cuatro bucles enumerados. En relación con ello, nuestra investigación analizó los mecanismos habituales de toma de decisiones durante el trabajo científico (gráfica 6). Cuando éstas se refieren a la adscripción a líneas de investigación, un $21.7 \%$ de las respuestas indica que se consensua. Este funcionamiento horizontal y poco jerárquico se ve confirmado por un $19.6 \%$ que afirma que se realiza por adscripción individual y voluntaria. Como contrapartida, un $17.4 \%$ de los participantes sostiene que es decisión del director del grupo.

Otras formas de decisión son el debate en reuniones, formales o no, y en juntas directivas. Aunque aparecen con menor frecuencia en los datos cuantitativos, los estudios de caso revelan como fundamental el consenso logrado durante las charlas informales. De hecho, en uno de los grupos analizados, el desayuno es cita obligada y ocasión de comunicación y negociación.

Con respecto a las vías de difusión de los resultados de las investigaciones (gráfica 7), un 41.3\% de las respuestas indica que obedece a la propuesta de cada miembro, consensuada con el resto, y un 19.6\% indica que es decisión autónoma de los investigadores. También se menciona que se realiza a propuesta del director y, en última instancia, de forma voluntaria.

Respecto a la distribución de las tareas y responsabilidades (gráfica 8), el 45.7\% de los participantes sostiene que se realiza a propuesta de cada miembro y mediante consenso, evidenciando nuevamente un funcionamiento participativo. En cambio, el 30.4\% de las respuestas afirma que se realiza por asignación del director. Finalmente, el 26.1\% sugiere la autoasignación. Seguramente la participación en la toma de estas decisiones varía entre los miembros de distintas categorías. Por otra parte, un 13\% de las respuestas de los investigadores afirma que existe rotación de tareas entre los miembros y, entre investigadores seniors, también de responsabilidades.

Siendo los encuentros entre los componentes del grupo ámbitos de negociación y toma de decisiones, su realización y contenido revisten interés. Preguntados al respecto, un 34.8\% de los investigadores afirma que las 


\section{Gráfica 6. Mecanismos habituales de toma de decisiones}

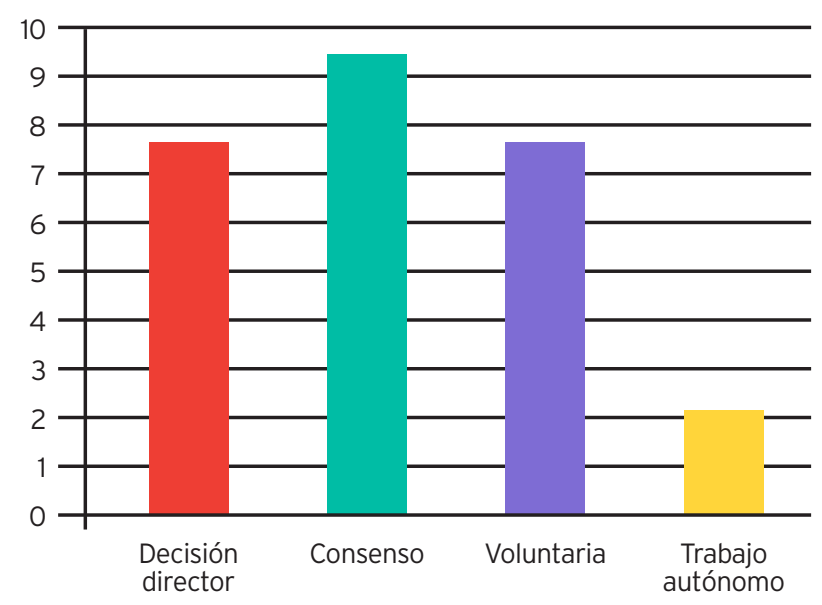

Gráfica 7. Difusión de resultados

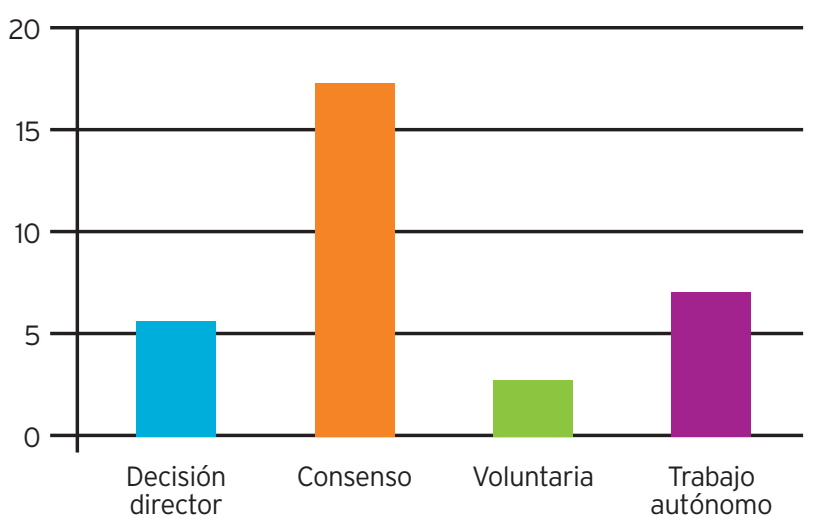

\section{Gráfica 8. Mecanismos habituales de distribución de tareas}

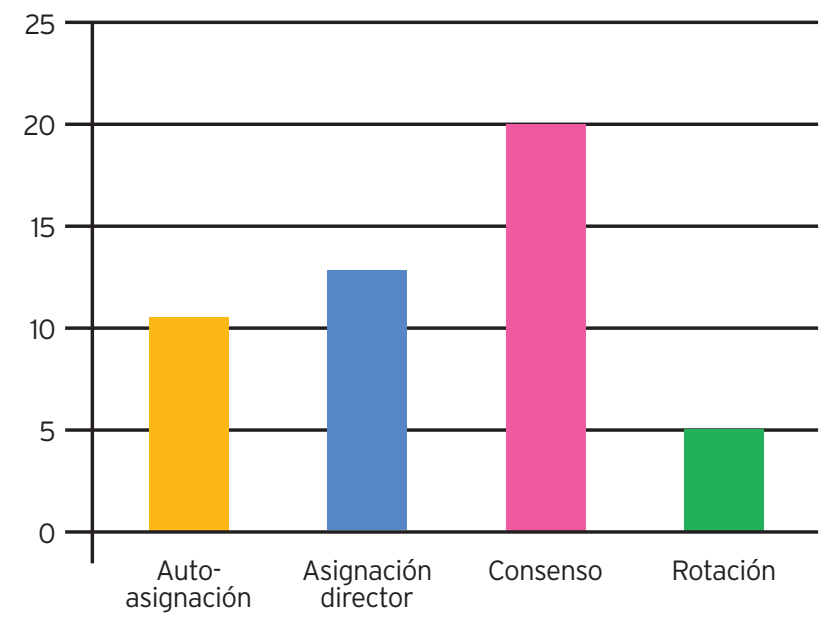

reuniones tienen lugar según las necesidades. Asimismo, sostienen que se convocan para realizar actividades de formación y un $32.6 \%$ indica que dedican reuniones a la distribución y gestión de recursos y de información sobre proyectos, mientras que el $26.1 \%$ de los encuestados afirma que se convocan reuniones de planificación de la tarea académica y de intercambio social.

Por otra parte, los grupos de investigación inscritos en universidades se reúnen con frecuencia por pertenecer al mismo departamento, lo que parece tener más peso en la definición del comportamiento y de las relaciones entre los miembros que la adscripción al grupo. Asimismo, los integrantes de los grupos cuentan con una autonomía que les permite perseguir metas no siempre compartidas. Esto se debe, en buena medida, a que carecen de mecanismos rigurosos, formalizados y efectivos de control, siendo elementos culturales y simbólicos los que los 
cohesionan. Puede, entonces, caracterizarse a los grupos de investigación como estructuras débilmente acopladas, al punto de que uno de los participantes afirma que lo que une al grupo de forma "nominal" es la ayuda oficial de la Junta de Andalucía. En esta línea, algunos miembros de uno de los grupos estudiados como caso - incluido su director - afirmaban desconocer la composición exacta de su grupo de investigación.

La frecuencia de las reuniones que mantienen los grupos responde — según los datos - a las necesidades que van surgiendo, aunque en la mayoría de ellos tienen carácter semanal y su convocatoria corre a cargo — según el 52.2\% de las respuestas - del director. Esto lleva a preguntarse por el papel que desempeña - más allá de las intenciones democráticas manifiestas - esta figura. Su relevancia no sorprende, en tanto coincide con la importancia que la literatura atribuye al líder en el impulso del funcionamiento grupal.

Por otra parte, según los datos, el estilo en la toma de decisiones tiende a fluctuar entre cierta centralización en el director del grupo - por ejemplo, la iniciativa de convocar a los miembros - y el predominio de mecanismos de consenso en la distribución de funciones y tareas. En síntesis, los grupos cuyos responsables han respondido al cuestionario adoptan, en opinión de éstos, un funcionamiento basado en el diálogo y el consenso más que en la autoridad, lo cual parece relacionarse con el interés por mantener valores que identifican al grupo y a su forma de trabajar.

Similar afán de integración se refleja en las acciones que, de manera sistemática, llevan a cabo los directores de los grupos participantes en el estudio de casos. Uno de ellos, por ejemplo, afirma estar especialmente atento a las manifestaciones de una de sus colaboradoras, particularmente sensible, y responder a ellas planteando directamente las cuestiones urticantes. Otra directora, en cambio, elige un estilo indirecto, basado en la clara distinción entre factores personales y profesionales. Probablemente esta actitud se oriente a contrarrestar el deterioro que los periodos de convivencia a los que el trabajo de campo obliga, pudieran generar en los vínculos.

Asimismo, el contenido de las reuniones se planifica según las necesidades, haciendo frente a eventuales dificultades cotidianas. Quizás como resultado de esta atención a las relaciones entre los miembros, en los datos no se identifican conflictos relevantes en el funcionamiento de los grupos como estructuras sociales.

\section{Conclusiones y prospectiva}

Empezaremos esta sección recordando el contraste entre la relativamente breve trayectoria formal de los grupos de investigación andaluces de excelencia y el largo tiempo que muchos de ellos llevan trabajando, incluso con similar composición, aunque sin reconocimiento por parte de la administración. Se trata, asimismo, de grupos estables en cuanto a su pertenencia organizativa y a la titularidad de su dirección, coincidiendo en esto con las descripciones de la literatura especializada (Rey-Rocha et al., 2006) que atribuye a la continuidad de los grupos una influencia positiva en la calidad de su labor científica.

Esta estabilidad puede relacionarse con que la constitución de los grupos suele tener lugar a partir de la reunión de jóvenes investigadores en torno de uno de prestigio y reconocida capacidad formativa. En este sentido, el mecanismo más frecuente de incorporación de nuevos miembros es la solicitud de los propios interesados, complementada por una colaboración previa. Figuras como las del alumno interno o del colaborador honorario - propias de las organizaciones universitarias, en las que se inscriben la mayoría de estos grupos - suelen constituir un primer paso en la carrera académica. Esta forma de colaboración con el grupo con fines formativos funciona como un "periodo de prueba", durante el cual el aspirante da muestras de su valía y tras el cual puede solicitar su plena incorporación. 
La conformación de los grupos en torno de una figura prestigiada condiciona, asimismo, la definición de una estructura jerarquizada. En ella, el liderazgo es ejercido por el/la director/a, quien ocupa una posición claramente diferenciada de la de los investigadores más jóvenes. A medida que éstos van adquiriendo experiencia y ganando espacio en el grupo, se delega en ellos algunas responsabilidades y tareas.

Por otra parte, cabe conjeturar que la estabilidad del grupo tiene un efecto positivo en lo psicosocial, favoreciendo el establecimiento de vínculos de mutuo apoyo que contrarrestan el estrés derivado de la competitividad y de la presión por el logro, incrementando la motivación derivada del compromiso con el conjunto.

Un elemento al que la literatura ha atribuido una infundada importancia - según nuestros datos y los de otros estudios recientes, como el de Horta y Lacy (2011) - como determinante de la calidad de la producción científica, es el tamaño de los grupos. En nuestra muestra, encontramos grupos relativamente pequeños en los que la representación de investigadores estables es variable. En cambio, hay coincidencia en cuanto a la asunción por parte de dichos investigadores de tareas de docencia y gestión, a diferencia de los investigadores invitados o no estables que sólo participan en pie de igualdad en la labor investigadora. También los miembros en formación restringen su actividad a esta última, probablemente, por estar elaborando su tesis doctoral.

Se aprecia, en definitiva, una clara distribución de roles y funciones entre los miembros de los grupos, garantía de los márgenes de acción de cada uno. Dado que cada uno sabe "cuál es su lugar" y lo acepta, las posibilidades de discordia son escasas y se facilita un funcionamiento basado en el consenso.

Esta misma certeza en la distribución de roles y responsabilidades legitima la toma de determinadas decisiones por parte del director del grupo - como la relativa a la asunción de tareas que requieren esfuerzos de escaso provecho inmediato pero son necesarias para la continuidad de la labor del grupo-, evitando el surgimiento de tensiones. Confirma esta idea el que no se identifiquen en los datos problemáticas relativas al funcionamiento de los grupos como sistemas sociales.

No obstante, siendo inevitable la existencia de roces en un grupo humano, cabe interpretar lo antedicho como un intento de situar fuera del grupo las fuentes de tensión, para librar al contexto interno de elementos potencialmente disruptivos. Este mecanismo - no necesariamente intencionado ni consciente - contribuiría a la cohesión del grupo frente a un entorno no siempre idóneo para la tarea científica, como el de la aplicación de medidas de restricción de fondos y endurecimiento del acceso a la carrera académica. Esto parecería justificar la importancia de prestar mayor atención en estudios futuros a los mecanismos por los que las dinámicas sociales de los grupos académicos condicionan su rendimiento científico, ya señalada por Hoffman y otros (2013).

Esta conjetura debería ser objeto de estudio más pormenorizado, al igual que otras relativas a las características intrínsecas a los miembros de los grupos como las variables de edad y de sexo de los miembros del grupo, o su formación de base- que no fueron atendidas en nuestro estudio. Entendemos que estas cuestiones pueden condicionar el desempeño individual y, en alguna medida, grupal. Asimismo, la indagación de la biografía de los miembros de estos grupos y de la manifestación de rasgos de personalidad que puedan correlacionar con la participación en grupos de excelencia - como la competitividad, la tolerancia al estrés y a la presión, etcétera- pueden proveer información valiosa para comprender el funcionamiento de los grupos científicos. La puesta en juego de estrategias e instrumentos como la reconstrucción de biografías académicas y el empleo de técnicas proyectivas, aportaría al entendimiento de cuestiones aquí planteadas y a la formulación de otras nuevas. 


\section{Referencias}

Becher, T. (2001), Tribus y territorios académicos, Barcelona, Gedisa Editorial.

Blasi, B. y S. Romagnosi (2012), "Social dynamics in scientific practices: focus on research groups", en Sociologia, vol. 46, núm. 2, pp. 66-77.

Blasi, B. y S. Romagnosi (2009), "Reflection on the collectivization of science through research groups", en Fournal of Science Comunication, vol. 8, núm. 4, pp. $1-4$.

Bleger, J. (1994), Psicohigiene y psicología institucional, Buenos Aires, Paidós.

Boardman, P. y E. A. Corley (2008), "University research centers and the composition of research collaborations", en Research Policy, vol. 37, pp. 900-913.

Bourdieu, P. (2007), Razones prácticas. Sobre la teoría de la acción, Barcelona, Anagrama.

Carayol, N.y M.Matt(2004), "Does research organization influence academic production? Laboratory level evidence from a large European university", en Research Policy, vol. 33, pp. 1081-1102.

Fernández Esquinas, M. y C. Torres Albero (2009), "La ciencia como institución social: clásicos y modernos institucionalismos en la sociología de la ciencia”, en Arbor, vol. 185, núm. 738, pp. 663-687

Etzkowitz, H. (1993), "Individual investigators and their research groups", en Minerva, vol. 30, pp. 28-50.

Hackett, E. J., D. Conz, J. Parker, J. Bashford y S. DeLay (2004), "Tokamaks and turbulence: research ensembles, policy and technoscientific work", en Research Policy, vol. 33, pp. 747-767.

Hemlin, S. (2006), "Creative knowledge environments for research groups in biotechnology. The influence of leadership and organizational support in universities and business companies", en Scientometrics, vol. 67, núm. 1, pp. 121-142.

Hoffman, D., B. Blasi, B. Culum, Z. Dragsic, A. Ewen, H. Horta, T. Nokkala y C. Rios-Aguilar (2013), "The methodological illumination of a blind spot: information and communication technology and international research team dynamics in a higher education research program", en Higher Education, DOI10.1007/s10734-013-9692-y

Horta, H. y T. A. Lacy (2011), "How does size matter for science? Exploring the effects of research unit size on academics' scientific productivity and information exchange behaviors", en Science and Public Policy, vol. 38, núm. 6, pp. 449-460.

Latour, B. (2001), La esperanza de Pandora, Barcelona, Gedisa.

Lucas, L. (2006), The research game in academic life, Londres, Open University Press.

Moretnd, R.L. (1987), "The formation of small groups", en C. Hendrick (ed.), Group Processes, Londres, Sege, pp. 80-110.

Prpic, K. (1994), "The socio-cognitive frameworks of scientific productivity", en Scientometrics, vol. 31, núm. 3, pp. 293-311.

Rey-Rocha,J.,M.J.Martín-SempereyJ.Sebastián(2008), "Estructura y dinámica de los grupos de investigación", en Arbor, vol. 184, núm. 732, pp. 743-757.

Rey-Rocha, J., B. Garzón-García y M. J. Martín-Sempere (2006), 'Scientists' performance and consolidation of research teams in Biology and Biomedicine at the Spanish Council for Scientific Research", en Scientometrics, vol. 69, núm. 2, pp. 183-212.

Rickards, T. y S. Moger (2000), "Creative leadership processes in project team development: an alternative to Tuckman's stage model", en British Fournal of Management, vol. 11, núm. 4, pp. 273-283.

Sundstrom, E., K. De Meuse y D. Futrell (1990), "Work teams applications and effectiveness", en American Psychologist, vol. 45, núm. 2, pp. 120-133.

Triadó Ivern, X. y P. Aparicio Chueca (2006), El perfil de los grupos de investigación más competitivos. Estudio del caso de la Universidad de Barcelona, Barcelona, AEDEM.

Tuckman, B. W. (1995), "Developmental Sequences in small groups", en Psychological Bullentin, vol. 63, núm. 6, pp. 384-963. 
Tuckman, B. W. y M. A. G. Jensen (1977), "Stages of smallgroup development revisited", en Group and Organization Studies, vol. 2, núm. 4, pp. 419-427.
Ziman, J. (1987), "The problem of 'problem choice'", en Minerva, vol. 25, núm. 1-2, pp. 92-106.

\section{Cómo citar este artículo:}

Altopiedi, Mariana, Elena Hernández-de la-Torre y Julián López-Yáñez (2015), “Características relevantes de grupos de investigación destacados en Andalucía", en Revista Iberoamericana de Educación Superior (RIES), México, UNAM-IISUE/Universia, vol. VI, núm. 16, pp. 126-142, https://ries.universia.net/article/view/1077/caracteristicas-relevantes-grupos-investigacion-destacados-andalucia [consulta: fecha de última consulta]. 